\title{
Thermodynamic geometry of Nambu-Jona Lasinio model
}

\author{
P. Castorina ${ }^{2,4}$, D. Lanteri ${ }^{1,2, a}{ }_{\mathbb{D}}$, S. Mancani $^{3}$ \\ ${ }_{1}^{1}$ Dipartimento di Fisica, Università di Catania, Via Santa Sofia 64, 95123 Catania, Italy \\ 2 INFN, Sezione di Catania, 95123 Catania, Italy \\ 3 Dipartimento di Fisica, Università di Roma "La Sapienza", Piazzale Aldo Moro 2, 00185 Rome, Italy \\ 4 Institute of Particle and Nuclear Physics, Faculty of Mathematics and Physics, Charles University, \\ V Holešovičkách 2, 18000 Prague 8, Czech Republic
}

Received: 24 July 2019 / Accepted: 20 December 2019 / Published online: 13 January 2020

(C) Società Italiana di Fisica (SIF) and Springer-Verlag GmbH Germany, part of Springer Nature 2020

\begin{abstract}
The formalism of Riemannian geometry is applied to study the phase transitions in Nambu-Jona Lasinio (NJL) model. Thermodynamic geometry reliably describes the phase diagram, both in the chiral limit and for finite quark masses. The different thermodynamic geometrical behavior of NJL model and of $(2+1)$ Quantum Chromodynamics at high temperature and small baryon density gives some hints on the connection between chiral symmetry restoration/breaking and deconfinement/confinement regimes.
\end{abstract}

\section{Introduction}

Geometry, and in particular differential geometry, is now considered a powerful tool to study statistical systems.

Indeed, information geometry [1-3], which started with the seminal paper by Rao [4] has emerged from studies of invariant geometrical structure involved in statistical inference. It defines a Riemannian metric together with dually coupled affine connections in a manifold of probability distributions.

These geometric structures play important roles not only in statistical inference but also in wider areas of information sciences, such as machine learning, signal processing, optimization, neuroscience, mathematics and, of course, physics [1-3].

Thermodynamic geometry (TG), a specific application of information geometry methods to equilibrium thermodynamics, started with an initial $[4,5]$ definition of a metric for statistical systems, i.e. a measure of the "distance" between different thermal equilibrium configurations, later refined in Ref. [6] by determining the metric tensor, $g_{\mu \nu}$, through the Hessian of the entropy density.

This definition of $g_{\mu \nu}$ is crucial since the resulting distance is in inverse relation with the fluctuation probability between equilibrium states and, moreover, it leads to the "interaction hypothesis", i.e. the correspondence between the absolute value of the scalar curvature $R$ (an intensive variable, with units of a volume, evaluated by the metric) and $\xi^{3}$, the cube of the correlation length, $\xi$, of the thermodynamic system. Indeed, a covariant and consistent

\footnotetext{
a e-mail: daniele.lanteri@ct.infn.it
} 
thermodynamic fluctuation theory can be developed [7], which generalizes the classical fluctuations theory and offers a theoretical justification to the physical meaning of $R$.

TG has been tested in many different systems: in phase coexistence for helium, hydrogen, neon and argon [8], for the Lennard-Jones fluids [9,10], for ferromagnetic systems and liquidliquid phase transitions [11]; in the liquid-gas-like first-order phase transition in dyonic charged AdS black hole [12]; in the Hawking-Page transitions in Gauss-Bonnet-AdS black holes [13].

More recently [14,15], TD has been applied to field theories and, in particular, to quantum chromodynamics (QCD) at large temperature and low baryon density, to evaluate the (pseudo-) critical deconfinement temperature $T_{\mathrm{c}}$ and to compare the results with the Hadron resonance gas models.

In this paper, a systematic application of TD to the Nambu-Jona-Lasinio (NJL) model is carried out. This study is not only interesting per se since the NJL model gives clear indications on some dynamical mechanism, as chiral symmetry, for low-energy QCD but also because a QCD fundamental property, quark confinement, is missing in NJL model with some interesting consequences on the geometrical description.

The TD approach is recalled in Sect. 2 and in Sect. 3 the phase diagram of the Nambu-Jona Lasinio model is discussed. Section 4 is devoted to the thermodynamic geometry description of chiral symmetry restoration in NJL model in the chiral limit and for finite fermion masses. The geometrical difference in describing QCD and NJL phase transitions is considered in Sects. 5 and 6 contains our comments and conclusions.

\section{Thermodynamic geometry}

In this section, the procedure to define the thermodynamic metric is briefly recalled (the details are in Ref. $[7,16]$ ) and the description of phase transitions by the scalar curvature, $R$, is discussed, making also use of the application to real fluids.

\subsection{Thermodynamic metric}

Let $A_{\mathrm{U}}$ be a large thermodynamic system (universe) and let us consider an open subsystem $A$ with thermodynamic coordinates $a^{0}$, the internal energy density, and $a^{i}$, the number densities of particles of different species. The probability density to find $A$ in the "point" $a=\left(a^{0}, a^{1}, \ldots\right)$ is given by

$$
P\left(a, a_{\mathrm{U}}\right) d^{n} a=C e^{S_{\mathrm{U}}\left(a, a_{\mathrm{U}}\right)} d^{n} a,
$$

being $C$ a normalization constant, $a_{\mathrm{U}}=\left(a_{\mathrm{U}}^{0}, a_{\mathrm{U}}^{i}, \ldots\right)$ denotes the state of the universe and $S_{\mathrm{U}}$ its total entropy, formally regarded as an exact function of the parameters of $A$ and $A_{\mathrm{U}}$.

On the basis of the maximum entropy principle and in the framework of Consistent and Covariant Fluctuation Theory (CCFT) [7], the thermodynamic properties of $A$ can be studied through the introduction of a quadratic form:

$$
(\Delta \ell)^{2}=g_{\mu \nu} \Delta a^{\mu} \Delta a^{\nu},
$$

where $\Delta a^{\mu}=a^{\mu}-a_{\mathrm{U}}^{\mu}$ and

$$
g_{\mu \nu}=-\left.\frac{\partial^{2} s}{\partial a^{\mu} \partial a^{\nu}}\right|_{a=a_{\mathrm{U}}}
$$


defines a positive-definite Riemannian metric on the space of thermodynamic states as the Hessian of the entropy density, $s$, with respect its natural variables $a^{\mu}$.

One can show [7] that previous formulas give the probability of the spontaneous fluctuations between equilibrium states. Indeed, by expanding Eq. (1) up to second order for $a \simeq a_{\mathrm{U}}$, the maximum entropy state, one finds the classical gaussian normalized fluctuation probability density:

$$
P\left(a, a_{\mathrm{U}}\right) d^{n} a=\left(\frac{V}{2 \pi}\right)^{\frac{n}{2}} \sqrt{g_{\mathrm{U}}} \exp \left\{-\frac{V}{2} g_{\mu \nu} \Delta a^{\mu} \Delta a^{\nu}\right\} d^{n} a,
$$

$g$ being the determinant of $g_{\mu \nu}$ and $\sqrt{g} d^{n} x$ the usual invariant volume on a Riemannian manifold.

In the analysis of the phase transitions in NJL model by thermodynamic geometry, we shall consider a two-dimensional manifold, where the intensive coordinates are $\beta=1 / T$ and $\gamma=-\mu / T$, with $\mu$ chemical potential. Moreover, the metric (3) turns out to be related to the derivatives of the potential $\phi=p / T$, where $p$ is the pressure [16]:

$$
g_{\mu \nu}=\left(\begin{array}{ll}
\phi, \beta \beta & \phi, \beta \gamma \\
\phi_{, \beta \gamma} & \phi_{, \gamma \gamma}
\end{array}\right)
$$

with the usual comma notation for derivatives.

The scalar curvature $R$ simply becomes

$$
R=\frac{1}{2 g^{2}}\left|\begin{array}{ccc}
\phi_{, \beta \beta} & \phi_{, \beta \gamma} & \phi_{, \gamma \gamma} \\
\phi_{, \beta \beta \beta} & \phi_{, \beta \beta \gamma} & \phi_{, \beta \gamma \gamma} \\
\phi_{, \beta \beta \gamma} & \phi_{, \beta \gamma \gamma} & \phi_{, \gamma \gamma \gamma}
\end{array}\right|
$$

\subsection{Phase transition in thermodynamic geometry}

The main results of the thermodynamic geometry within Ruppeiner's formulation [7] are (1) the (inverse) relation between the line element and the fluctuation probability between equilibrium states; (2) the, so-called, Interaction hypothesis: the absolute value of the scalar curvature $R$ is proportional to a power of the correlation length, i.e. $|R| \sim \xi^{d}$, where $d$ is the effective spatial dimension of the underling thermodynamic system.

The meaning of the correlation length and of the scalar curvature can be represented as in Fig. 1 (a schematic picture due to Widom [17]): the intricate line represents what the surface of density $\rho(r)=\rho_{0}$ might look at any instant. This surface separates two sides with local mean densities $\rho>\rho_{0}$ and $\rho<\rho_{0}$. By tracing any straight line, the intersection points with the surface $\rho_{0}$ are separated by an average distance equal to $\xi$. Because such points are separated by the same mean distance $\xi$, whatever the direction of the line, it is convenient to think that regions as volume elements ("droplets") of dimension $R \sim \xi^{d}$. Figure 2 shows a schematic summary of different configurations.

The interaction hypothesis has been confirmed by the study of the classical ideal gas ( $R=0$ [6]) and of the van der Waals gas [7], for which, near the liquid-vapor critical point, $T_{\mathrm{c}}$, the curvature is $R \sim\left|\left(T-T_{\mathrm{c}}\right) / T_{\mathrm{c}}\right|^{-2}$.

Other confirmations come from the study of the Takahashi Gas [7], the Curie-Weiss model [19], the ferromagnetic monodimensional Ising model [20]. For a more complete list of applications see Tab. I of Ref. [21].

The relation between $|R|$ and $\xi^{d}$ is easy to verify for second-order phase transitions, since $R$ diverges, but the criterium to define a new phase in term of the curvature $R$ for a first-order phase transition or a crossover is less clear. 
Fig. 1 Schematic picture of the meaning of $\xi$ : the intricate line represents the surface of $\rho(r)=\rho_{0}$, i.e., that separating two sides with local mean densities $\rho>\rho_{0}$ and $\rho<\rho_{0}$. By tracing any straight line, the intersection points are separated by an average distance equal to $\xi$. Figure from Ref. [18]
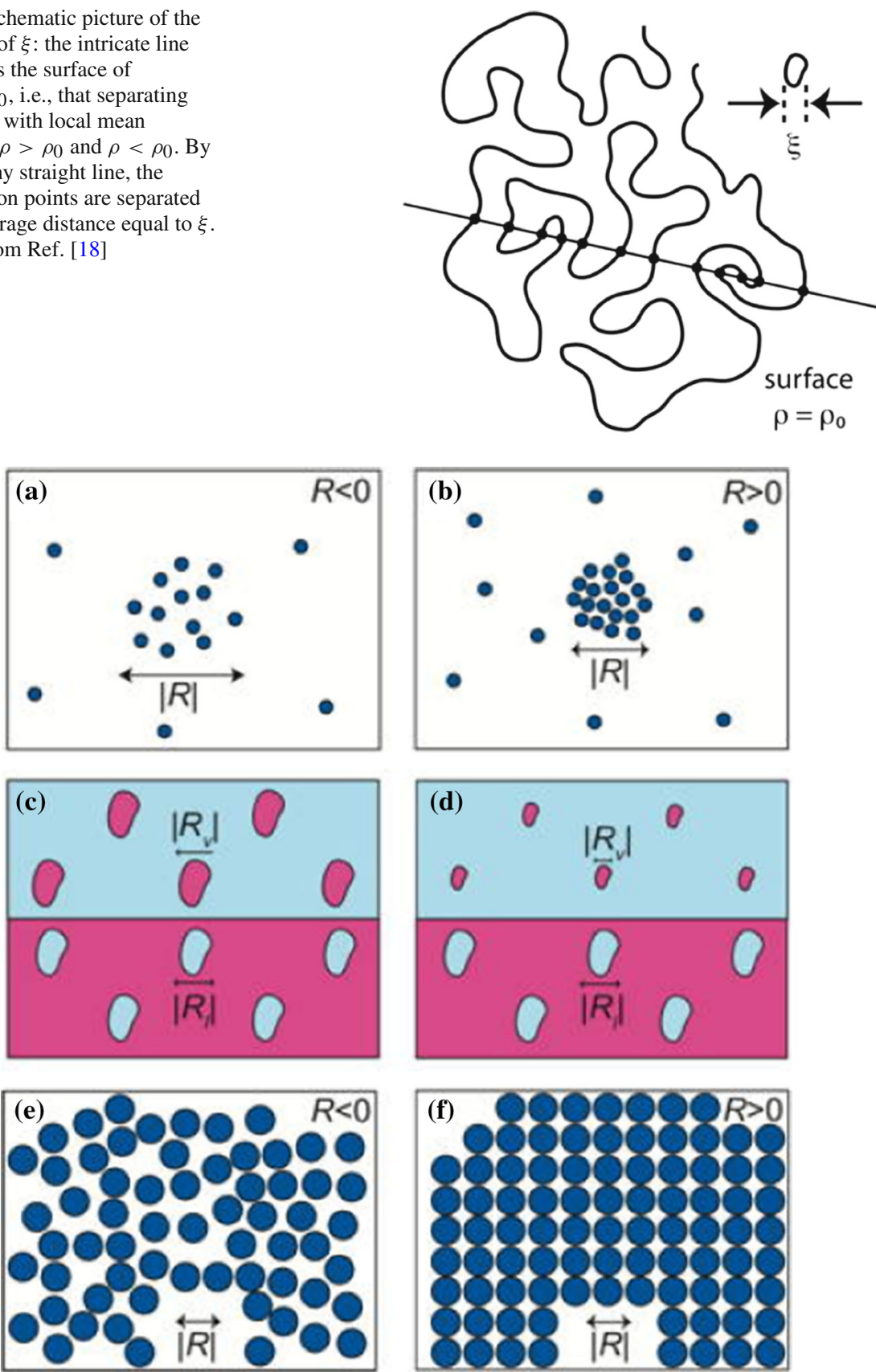

Fig. 2 Schematic pictures of different possible particle arrangements: a cluster of particles with volume $|R|$ pulled together by the attractive part of the interparticle interaction $(R<0)$; $\mathbf{b}$ a repulsive solid-like cluster held up by hard-core particle repulsion $(R>0)$; $\mathbf{c}, \mathbf{d}$ a fluid in two phases near the critical point: the bottom half is a liquid phase containing vapor droplets with volume $\left|R_{l}\right|$. The top half is a coexisting vapor phase containing liquid droplets with volume $\left|R_{\mathrm{V}}\right|$. In $\mathbf{c}\left|R_{\mathrm{V}}\right|=\left|R_{\mathrm{l}}\right|$ and the droplets are commensurate, in $\mathbf{d}$ liquid and vapor phases have incommensurate droplets; e liquid phase; $\mathbf{f}$ solid phase with $R>0$. Figure from Ref. [18] 
The approach called $R$-Crossing Method (RCM) [8] is often applied to define first-order phase transitions. It is based on the continuity of the scalar curvature: knowing the thermodynamic quantities in the two phases, i.e. $R$, one can build up the transition curve by imposing the continuity of $R$. The RCM, coherent with Widom's microscopic description of the liquid-gas coexistence region (i.e., with the idea that the correlation lengths of the two phases must be the same at the transition) has been tested in systems with different features: vapor-liquid coexistence line for the Lennard-Jones fluids [9,10], first- and second-order phase transitions of mean-field Curie-Weiss model (ferromagnetic systems), liquid-liquid phase transitions [11], phase transitions of cosmological interest as the liquid-gas-like firstorder phase transition in dyonic charged AdS black hole [12]. Another criterion applied in the study of first-order phase transitions in real fluids [18] and Lennard-Jones systems [10] is a first kind discontinuity in $R$.

Finally, two different phases can be linked by a crossover, as for the QCD deconfinement transition. Also in this case, there is no definitive conclusion on the behavior of $R$, although it has been recently shown [14] that the condition $R=0$ predicts a temperature for the transition from QCD to the Hadron resonance Gas at low baryon density in agreement with freeze out curve [22-24] and (within 10\%) with lattice data [25,26].

Another interesting aspect of the geometrical approach to phase transitions is that the sign of the scalar curvature brings information on the microscopic interactions since $R$ turns out to be positive for fermi statistical interactions and negative in the bosonic case $[27,28]$. Therefore, a change in sign of $R$ is an indication of the balance between effective interactions, even when no transition occurs, and theoretical curves with $R=0$ in pure fluids identify some anomalous behaviors observed in the experimental data of several substances (in particular, water) $[18,29]$. A transition from $R>0$ to $R<0$ has been also shown for the LennardJones system [9,10] and Anyon gas [30,31]. For black holes [32], the change in sign of the curvature occurs at the Hawking-Page transition temperature, therefore, associated with the condition $R=0$.

In the next sections, we shall apply the thermodynamic geometry approach to NJL phase diagram both in the chiral limit and for finite fermion mass. The behavior of the scalar curvature in the quantitative description of the critical line in the $T-\mu$ plane will be pointed out.

\subsection{An example: real fluids}

The geometrical study of fluids is based on the Helmholtz free energy per volume, $f$, in terms of $(T, \rho)$ coordinates ( $T$ is the temperature, $\rho=N / V$ is the particle density) and the corresponding thermodynamic line element is given by [18]

$$
\Delta \ell^{2}=-\frac{1}{T}\left(\frac{\partial^{2} f}{\partial T^{2}}\right)_{\rho} \Delta T^{2}+\frac{1}{T}\left(\frac{\partial^{2} f}{\partial \rho^{2}}\right)_{T} \Delta \rho^{2} .
$$

The scalar curvature turns out to be

$$
R=\frac{1}{\sqrt{g}}\left[\frac{\partial}{\partial T}\left(\frac{1}{\sqrt{g}} \frac{\partial g_{\rho \rho}}{\partial T}\right)+\frac{\partial}{\partial \rho}\left(\frac{1}{\sqrt{g}} \frac{\partial g_{T T}}{\partial \rho}\right)\right]
$$

with

$$
g_{T T}=-\frac{1}{T}\left(\frac{\partial^{2} f}{\partial T^{2}}\right)_{\rho}, \quad g_{\rho \rho}=\frac{1}{T}\left(\frac{\partial^{2} f}{\partial \rho^{2}}\right)_{T}
$$

and $g=g_{T T} g_{\rho \rho}$. 
In Refs. [18,29,33], the real fluid free energy is modeled on the NIST Chemistry WebBook and $R$ is evaluated in the liquid and vapor phases and along the liquid-vapor coexistence curve ending at the critical point $T_{\mathrm{c}}$.

At the critical point $R \rightarrow-\infty$ with a power law behavior and in the asymptotic critical region, i.e., very close to the critical temperature, the values of the scalar curvature evaluated in the two phases coincide. However, in other regions of the thermodynamic parameter space, the values of $R$ in the liquid and the vapor phases [18] are quite different and mesoscopic fluctuating structures of different sizes occur in the two phases (see Fig. 2d).

In the phase diagram of fluids, $R$ is generally found to be negative since the average molecular distances are such that the attractive part of the intermolecular potential dominates. However, different anomalous regions, i.e., with $R>0$, exist (see fig. 4 in Ref. [29]). They are localized (a) in the supercritical liquid region, near the melting line; (b) in the liquid phase near the triple point (for water); (c) in the vapor phase, in some regions called "repulsive clusters" [29].

The thermodynamic states for cases (a) and (b), named solid-like liquid states, emerge when the liquid organizes into solid-like structures at large densities, with a small intermolecular average separation. The states in "repulsive cluster" areas (case c) are characterized by values of $R$ much larger than the volume of a single molecule and by low density and have been observed in 97 different fluids (except those consisting of the simplest molecules) along the saturated vapor phase curve.

\section{Nambu-Jona Lasinio model}

In Nambu-Jona Lasinio (NJL) model with two flavors $(f=u, d)$, the $S U(2)$ Lagrangian [34-36] is given by

$$
\mathcal{L}_{S U(2)}=\bar{\psi}_{f}(i \not \partial-m) \psi_{f}+G\left[\left(\bar{\psi}_{f} \psi_{f}\right)^{2}+\left(\bar{\psi}_{f} i \gamma_{5} \vec{\tau} \psi_{f}\right)^{2}\right],
$$

being $G$ a dimensionful coupling, $m$ the current quark mass ( $m=0$ is the chiral limit) and $\vec{\tau}$ the Pauli matrices. In mean-field approximation the thermodynamic potential, $\Omega$, at finite temperature and chemical potential turns out to be [36]

$$
\Omega\left(M_{f}\right)=\frac{\left(M_{f}-m\right)^{2}}{4 G}+N_{\mathrm{f}} \Omega_{f},
$$

with

$$
\begin{aligned}
\Omega_{f}= & -2 N_{\mathrm{c}} \int \frac{\mathrm{d}^{3} p}{(2 \pi)^{3}} E_{f}-2 N_{\mathrm{c}} T \int \frac{\mathrm{d}^{3} p}{(2 \pi)^{3}} \ln \left[1+e^{-\frac{E_{f}+\mu_{f}}{T}}\right] \\
& -2 N_{\mathrm{c}} T \int \frac{\mathrm{d}^{3} p}{(2 \pi)^{3}}\left[1+e^{-\frac{E_{f}-\mu_{f}}{T}}\right],
\end{aligned}
$$

where $M_{f}$ is the dynamically generated mass, $E_{f}=\sqrt{p^{2}+M_{f}^{2}}, N_{\mathrm{c}}$ and $N_{\mathrm{f}}$ are the number of colors and flavors, respectively, $\mu_{f}$ is the quark $f$ chemical potential and the integrals are regulated by a cutoff $\Lambda$. For $m_{u}=m_{d}, \mu=\mu_{u}=\mu_{d}$, the generated quark mass is $M=M_{u}=M_{d}$.

To evaluate the minimum of $\Omega$ by Eq. (11), one has to solve the self-consistent gap equation

$$
M=m-2 G\langle\bar{\psi} \psi\rangle,
$$


where $\langle\bar{\psi} \psi\rangle$ is the quark-antiquark condensate:

$$
\langle\bar{\psi} \psi\rangle=-2 N_{\mathrm{c}} N_{\mathrm{f}} \int \frac{\mathrm{d}^{3} p}{(2 \pi)^{3}} \frac{M}{E} \Psi(T, \mu)
$$

with

$$
\Psi(T, \mu)=1-n_{+}(\mu)-n_{-}(\mu)
$$

and

$$
n_{ \pm}(\mu)=\frac{1}{1+\exp \left\{\frac{E \pm \mu}{T}\right\}}
$$

For three flavors with $m_{u}=m_{d}=m$, and $m_{s} \neq m$, one has $M_{u}=M_{d} \neq M_{s}$, and the $S U$ (3) Lagrangian is [36]

$$
\mathcal{L}_{S U(3)}=\bar{\psi}(i \not \partial-\widehat{m}) \psi+\mathcal{L}_{4}+\mathcal{L}_{6},
$$

where

$$
\mathcal{L}_{4}=G \sum_{a}\left[\left(\bar{\psi} \lambda_{a} \psi\right)^{2}+\left(\bar{\psi} i \gamma_{5} \lambda_{a} \psi\right)^{2}\right]
$$

and the 't Hooft interaction, $\mathcal{L}_{6}$, is given by

$$
\mathcal{L}_{6}=-K\left[\operatorname{det} \bar{\psi}\left(1+\gamma_{5}\right) \psi+\operatorname{det} \bar{\psi}\left(1-\gamma_{5}\right) \psi\right]
$$

with $\psi=(u, d, s)^{T}, \widehat{m}=\operatorname{diag}\left(m, m, m_{s}\right), \lambda_{0}=\sqrt{2 / 3} \mathbb{1}_{3 \times 3}$, being $\mathbb{1}_{3 \times 3}$ the $3 \times 3$ identity matrix, and where $\lambda_{a}(a=1, \ldots, 8)$ are the Gell-Mann matrices and $K$ and $G$ dimensionful couplings.

The gap equations,

$$
M_{i}=m_{i}-4 G\left\langle\bar{\psi}_{i} \psi_{i}\right\rangle+2 K\left\langle\bar{\psi}_{j} \psi_{j}\right\rangle\left\langle\bar{\psi}_{k} \psi_{k}\right\rangle \quad(j, k \neq i),
$$

are coupled with the quark condensates

$$
\left\langle\bar{\psi}_{i} \psi_{i}\right\rangle=-2 N_{\mathrm{c}} \int \frac{\mathrm{d}^{3} p}{(2 \pi)^{3}} \frac{M_{i}}{E_{i}} \Psi_{i}
$$

where

$$
\Psi_{i}=1-\frac{1}{1+e^{\frac{E_{i}+\mu_{i}}{T}}}-\frac{1}{1+e^{\frac{E_{i}-\mu_{i}}{T}}},
$$

and the mean-field thermodynamic potential $\Omega$ turns out to be [36]

$$
\Omega=\sum_{f=u, d, s} \Omega_{f}+2 G \sum_{f=u, d, s}\left\langle\bar{\psi}_{f} \psi_{f}\right\rangle^{2}-4 K\langle\bar{u} u\rangle\langle\bar{d} d\rangle\langle\bar{s} s\rangle,
$$

with $\Omega_{f}$ in Eq. (12).

Finally, the potential we need for the thermodynamic geometry calculations is

$$
\phi(\beta, \gamma)=\frac{P}{T}=-\Omega(\beta, \gamma) \beta,
$$

where $P=-\Omega$ is the pressure. 

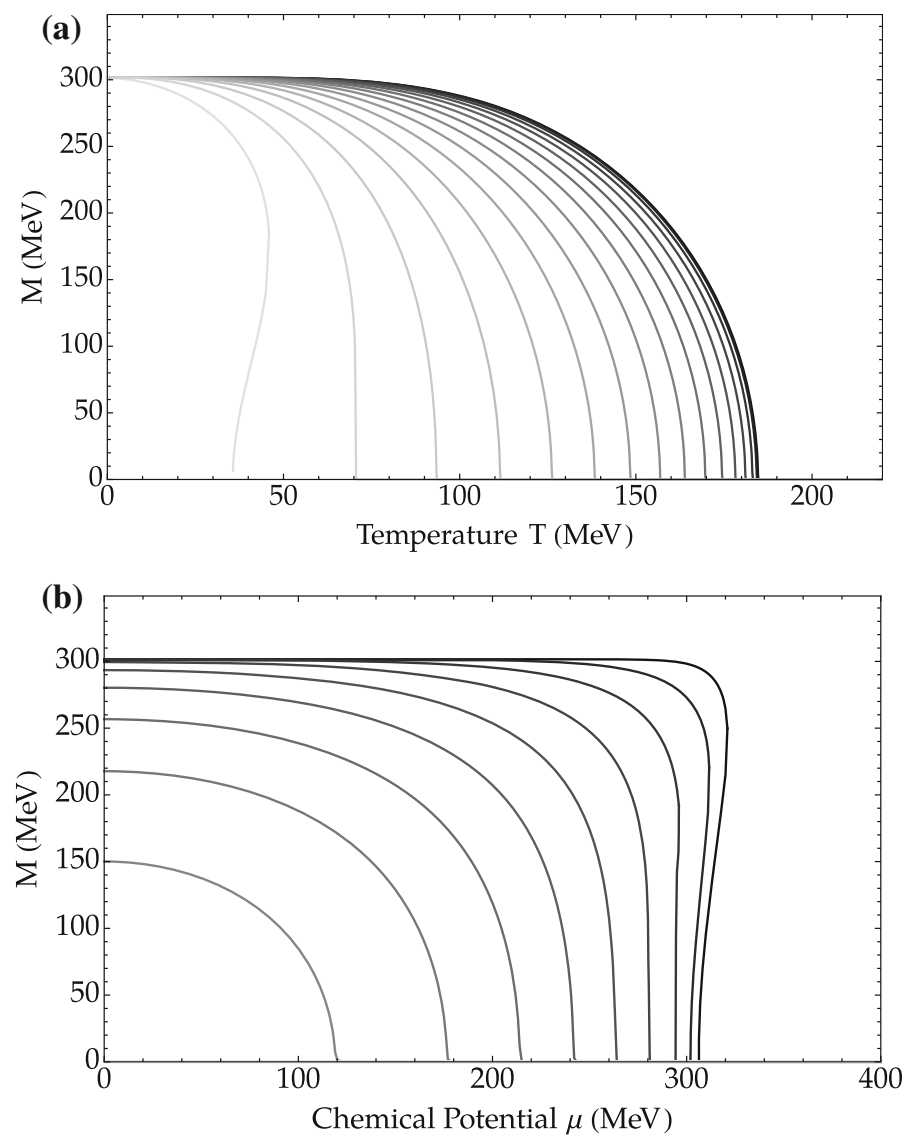

Fig. 3 a The dynamically generated mass, $M$, in the NJL model with two flavors in the chiral limit $\left(m_{u}=\right.$ $m_{d}=0 \mathrm{MeV}$ ) and again the temperature. Black line is for $\mu=0 \mathrm{MeV}$; the others are for growing $\mu$, up to $\mu=300 \mathrm{MeV}$ and with step of $\Delta \mu=20 \mathrm{MeV}$. $\mathbf{b} M$ as a function of the chemical potential $\mu$. Black line is for $T=10 \mathrm{MeV}$; the others are for growing $T$, up to $T=170 \mathrm{MeV}$ and with step of $\Delta T=20 \mathrm{MeV}$

\section{Thermodynamic geometry of chiral symmetry restoration in NJL model}

\subsection{Two flavors in the chiral limit}

Let us first discuss the chiral limit $(m=0)$ for two flavors, starting from the breaking of chiral symmetry at $T=\mu=0$, with the value of the dynamical mass $M_{0}(0,0)=300 \mathrm{MeV}$, corresponding to $\Lambda=650 \mathrm{MeV}$ and $G=5.01 \times 10^{-6} \mathrm{MeV}^{-2}[34,35]$.

The well-known solution $M(T, \mu)$ of the gap equation (13), for different values of the temperature and of the quark chemical potential, is plotted in Fig. 3a, b. The restoration of the chiral symmetry is a first-order phase transition at large chemical potential and a second-order one at low $\mu$.

The study of the critical line of the symmetry restoration, $T(\mu)$, by thermodynamic geometry requires the, straightforward but laborious, calculation of the scalar curvature $R$, reported in Appendix A. 


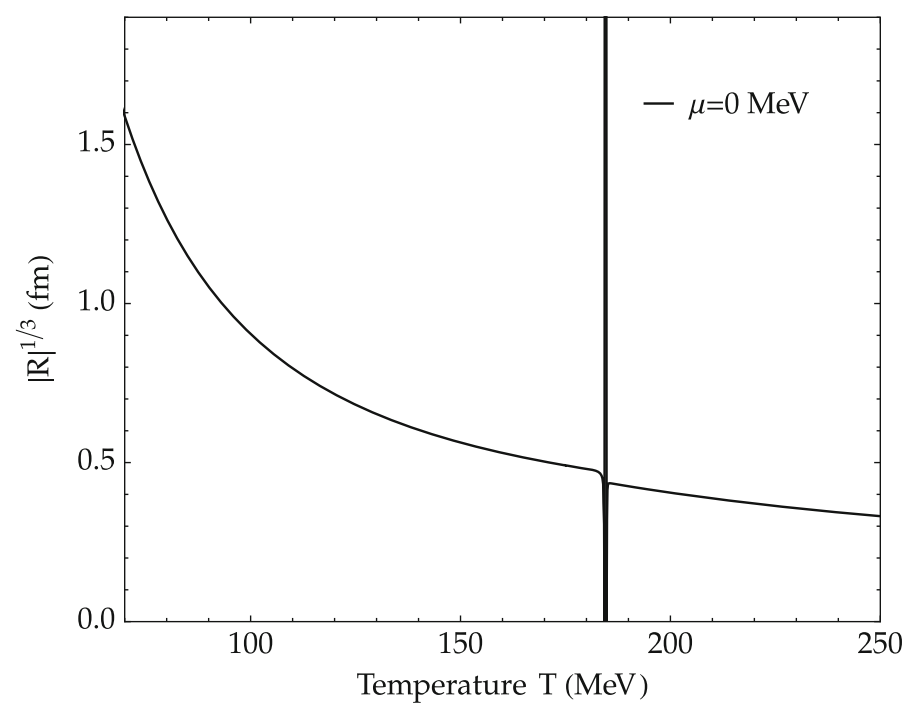

Fig. $4 R$ from $\mu=0 \mathrm{MeV}$ : second-order phase transition

It turns out that $|R|$ diverges at the critical temperature, i.e., there is a second-order phase transition, for $\mu<\mu^{\star} \simeq 290 \mathrm{MeV}$, as shown in Fig. 4 for $\mu=0$. For $\mu>\mu^{\star}$ there is, instead, a first-order phase transition. The dynamically generated mass, $M$, now takes the characteristic behavior plotted in Fig. 5, where the black curves (both the continuous and the dotted) are for $T=30 \mathrm{MeV}$ and the two light-gray lines define the spinodal points. Between the two spinodal (light-gray) lines one can evaluate three different scalar curvatures: the first one for the higher mass branch (black curve in Fig. 5); the second one for $M=0 \mathrm{MeV}$ and the last one is related to the $M$-branch that interpolates between $M=0$ and the upper $M$-curve (dotted curve in Fig. 5). At fixed temperature and between the spinodal lines (see Fig. 5), there is a discontinuity in $|R|$ which identifies the two dashed curves in Fig. 6.

The crossing temperature from the first-order phase transition to the second-order turns out to be about $58 \mathrm{MeV}$.

For small $\mu$ and near the transition, the curvature is negative, i.e., the interaction is mostly attractive, suggesting that the chiral symmetry restoration is due to thermal fluctuations.

On the other hand, at large chemical potential, $R$ turns out to be positive, indicating a screening of the potential.

The complete critical line obtained by thermodynamic geometry is depicted in Fig. 6 where the continuous line shows the second-order phase transition and the dashed lines the spinodal curves of the first-order one. The green band is the region of negative $R$.

\subsection{Two flavors with chiral masses}

With finite chiral quark masses, at high temperature and low chemical potential, there is a smooth crossover rather than a second-order phase transition. Moreover, the first-order phase boundary ends in a second-order endpoint [36].

The solution of the gap equation (13) (with $\Lambda=650 \mathrm{MeV}$ and $G=5.01 \times 10^{-6} \mathrm{MeV}^{-2}$ and $m_{0}=5.5 \mathrm{MeV}$ ) as a function of $T$ and $\mu$ is shown in Fig. 7a, b. 


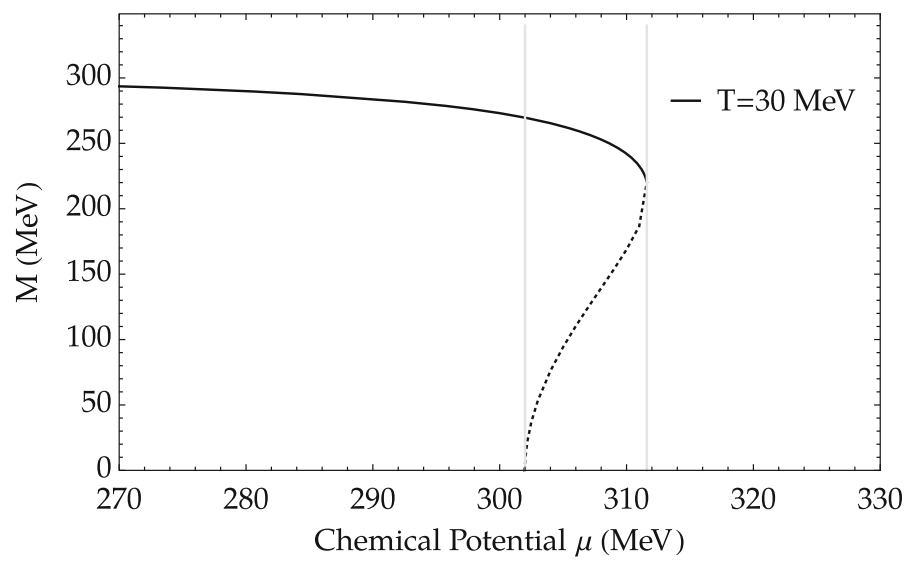

Fig. 5 The dynamically generated mass $M$ in the 2 flavors NJL chiral model and temperature $T=30 \mathrm{MeV}$

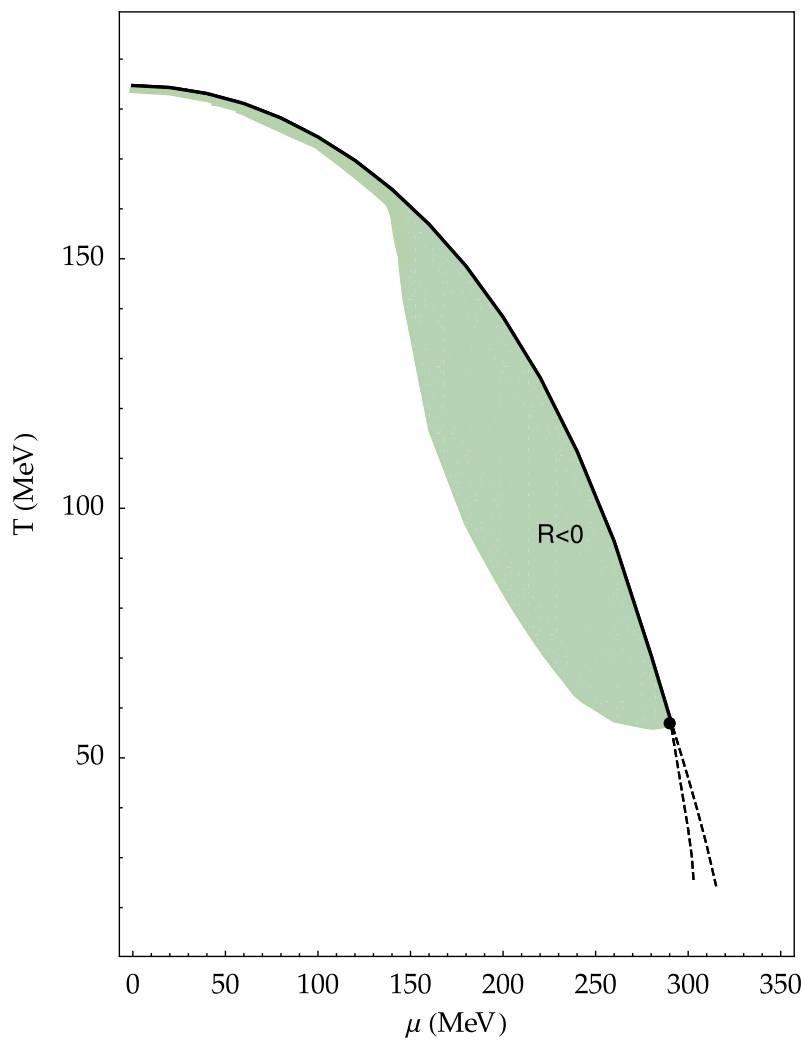

Fig. 6 The transition temperature: continuous line is for second-order phase transition and the dashed ones for the first-order one. The transition point is at $\mu_{\chi}^{\star}=290 \mathrm{MeV}$ and $T_{\chi}^{\star}=58 \mathrm{MeV}$. The green band is the region of $R<0$ 

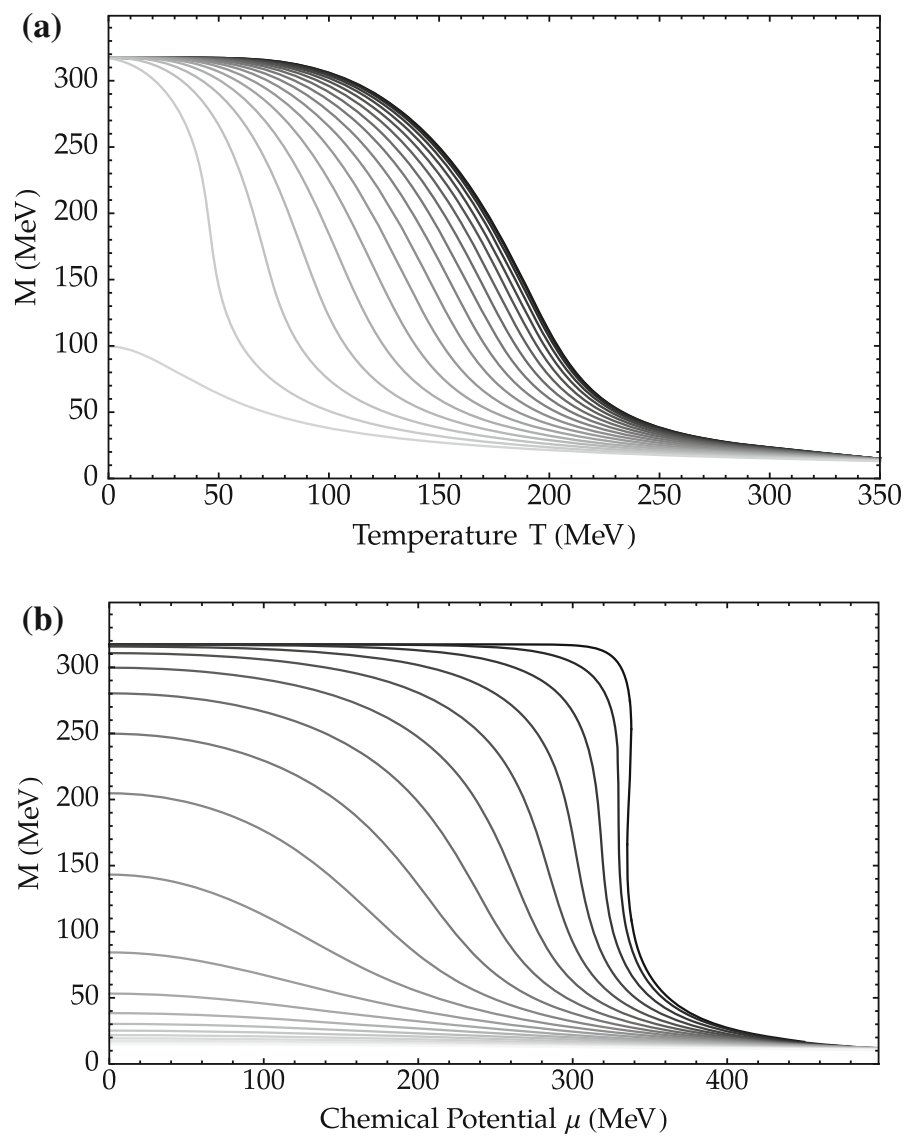

Fig. 7 a The dynamical generated mass, $M$, in the NJL model with two flavors of identical mass $\left(m_{u}=\right.$ $m_{d}=5.5 \mathrm{MeV}$ ) and again the temperature. Black line is for $\mu=0 \mathrm{MeV}$; the others are for growing $\mu$, up to $\mu=340 \mathrm{MeV}$ and with step of $\Delta \mu=20 \mathrm{MeV}$. b $M$ as a function of the chemical potential $\mu$. Black line is for $T=10 \mathrm{MeV}$; the others are for growing $T$, up to $T=400 \mathrm{MeV}$ and with step of $\Delta T=20 \mathrm{MeV}$

To clarify the effect of the chiral mass in the calculation of the scalar curvature, Fig. 8 shows that $R$ diverges in the chiral limit but for $m_{0} \neq 0$, near the transition temperature, it has a minimum, corresponding to a maximum of $|R|$, i.e., to a finite correlation length. Therefore, $m_{0} \neq 0$ changes the behavior of $R$ near the critical temperature: the divergence of the second-order phase transition turns into a minimum in the negative $R$ region and the transition temperature evaluated by the maximum of $|R|$ is completely in agreement with that one obtained by chiral susceptibility (see Eq. (48) in Appendix A).

For low temperature and large chemical potential, the scalar curvature $R$ has the same behavior previously discussed in the chiral limit, i.e., a first-order phase transition.

The critical point, $\left(T^{\star}, \mu^{\star}\right)$ between the crossover and the first-order phase transition depends on $m_{0}$ and for (the generally accepted value) $m_{0}=5.5 \mathrm{MeV}$ one has $\mu^{\star} \simeq 329 \mathrm{MeV}$ and $T^{\star} \sim 32 \mathrm{MeV}$.

In Fig. 9 are shown the metric determinant at the transition temperature (evaluated as the maximum of $|R|)$ and as a function of $\mu$, normalized to the value at $\mu=0,\left|g_{0}\right|$, and for two different mass $m_{0}$ (i.e., for crossover): solid line is for $m_{0}=5.5 \mathrm{MeV}$, the dotted 


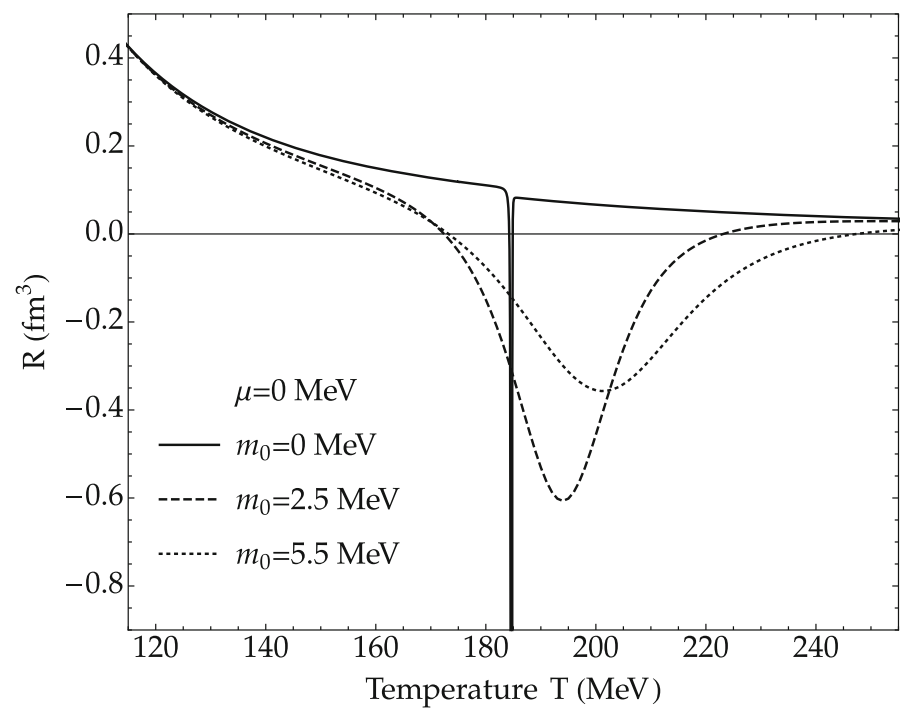

Fig. $8 R$ from $\mu=0 \mathrm{MeV}$ and different values of the bare mass $m_{0}$ : continuous line is from $m_{0}=0 \mathrm{MeV}$ (the chiral limit) and $R$ shows a negative divergence. Dashed line is from $m_{0}=2.5 \mathrm{MeV}$ and the dotted from $m_{0}=5.5 \mathrm{MeV}$; both show a finite region with negative $R$ around the transition temperature, which corresponds to the local maximum of $|R|$

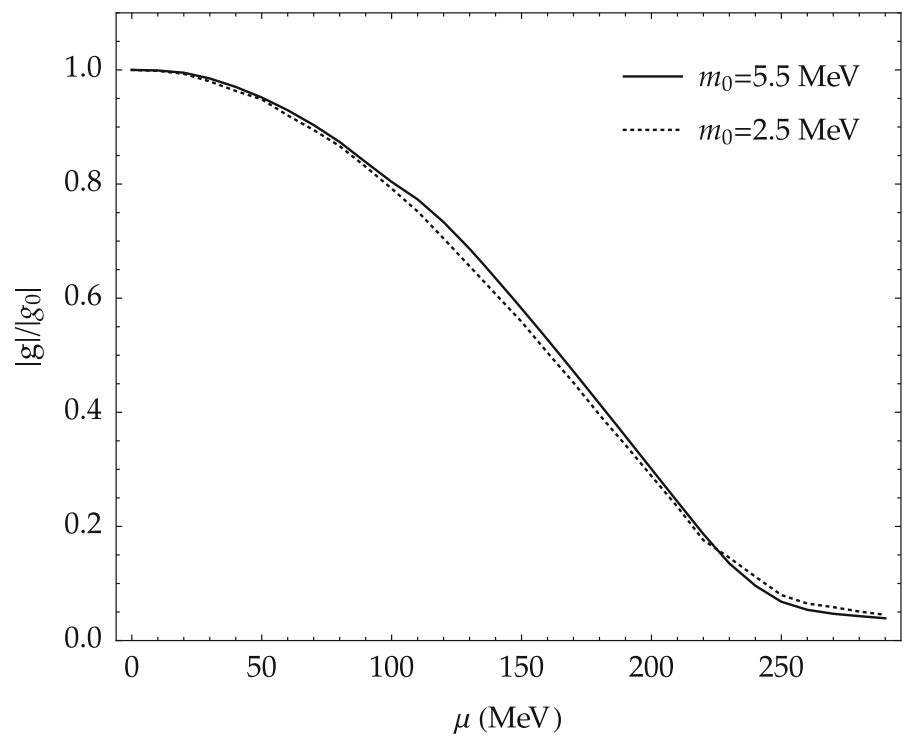

Fig. 9 Determinant of the metric at the transition temperature (evaluated as the maximum of $|R|$ ) and as a function of $\mu$, normalized to the value at $\mu=0,\left|g_{0}\right|$. Solid line is for $m_{0}=5.5 \mathrm{MeV}$, the dotted one for $m_{0}=2.5 \mathrm{MeV} .\left|g_{0}\left(m_{0}=5.5\right)\right|=2.010^{19} \mathrm{MeV}^{8}$ and $\left|g_{0}\left(m_{0}=2.5\right)\right|=1.810^{19} \mathrm{MeV}^{8}$ 


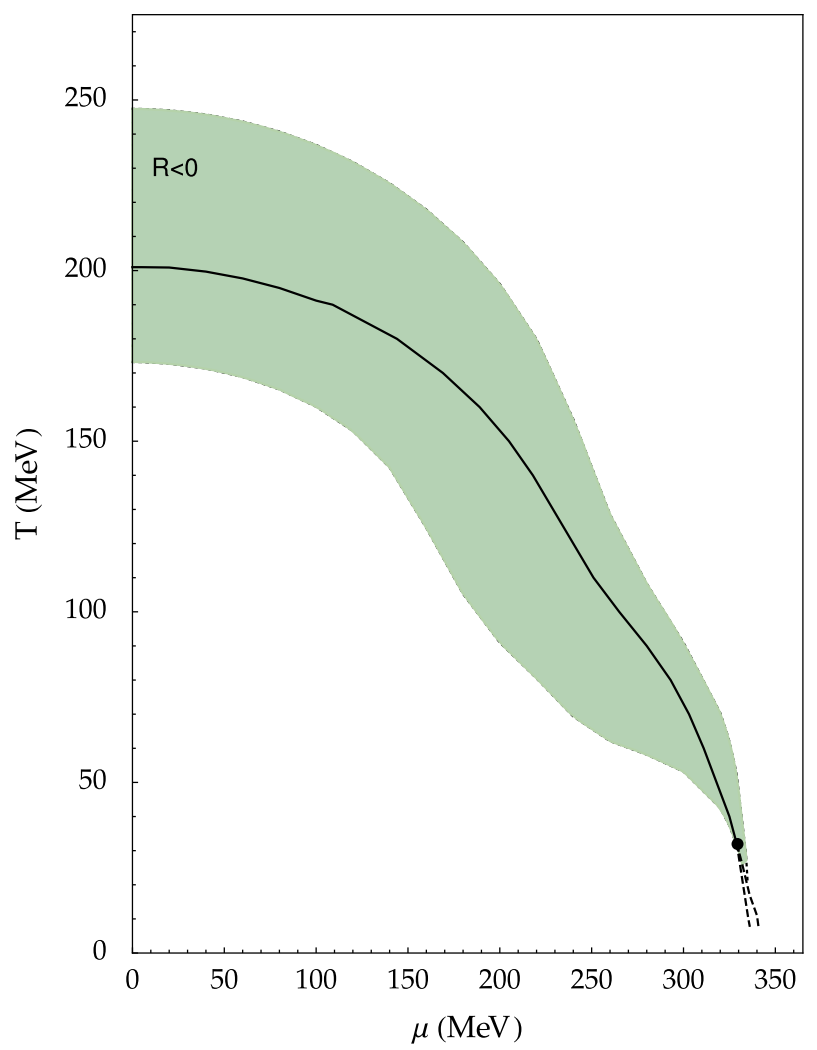

Fig. 10 The transition temperature by the $R$ conditions and from $m_{0}=5.5 \mathrm{MeV}$ : continuous line is obtained by the local maximum of $|R|$, the dashed ones indicate the spinodal lines. The circle is at $\mu^{\star}=329 \mathrm{MeV}$ and $T^{\star}=32 \mathrm{MeV}$. The green band is the region of $R<0$

one for $m_{0}=2.5 \mathrm{MeV}$. The values of $g_{0}$ are $\left|g_{0}\left(m_{0}=5.5\right)\right|=2.010^{19} \mathrm{MeV}^{8}$ and $\left|g_{0}\left(m_{0}=2.5\right)\right|=1.810^{19} \mathrm{MeV}^{8}$.

Figure 10 shows the critical line for $m_{0}=5.5 \mathrm{MeV}$ : the continuous line is obtained by the maximum of $|R|$ and the dashed ones are the spinodal curves. The black circle is at $\mu^{\star}=329 \mathrm{MeV}$ and $T^{\star}=32 \mathrm{MeV}$. The green band is the region of $R<0$.

\subsection{Three flavors}

Three flavor NJL model is studied with the parameter values [37]

$$
\begin{aligned}
& \Lambda=631.4 \mathrm{MeV}, \quad G \Lambda^{2}=1.835, \quad K \Lambda^{5}=9.29, \\
& m=5.5 \mathrm{MeV}, \quad m_{s}=135.7 \mathrm{MeV}
\end{aligned}
$$

and only one chemical potential $\left(\mu=\mu_{d}=\mu_{u}, \mu_{s}=0\right)$. The dynamically generated masses $M_{u}=M_{d}$ and $M_{s}$ are now solutions of the system of Eqs. (20) and (21). Their behavior is similar to that one depicted in Fig. 7, but with different values for light and strange quarks. Also in this case there is a crossover at low chemical potential and large $T$ and a first-order phase transition at low temperature and large $\mu$. The behavior of the scalar curvature is essentially the same of the previous case with two flavors and physical masses. 


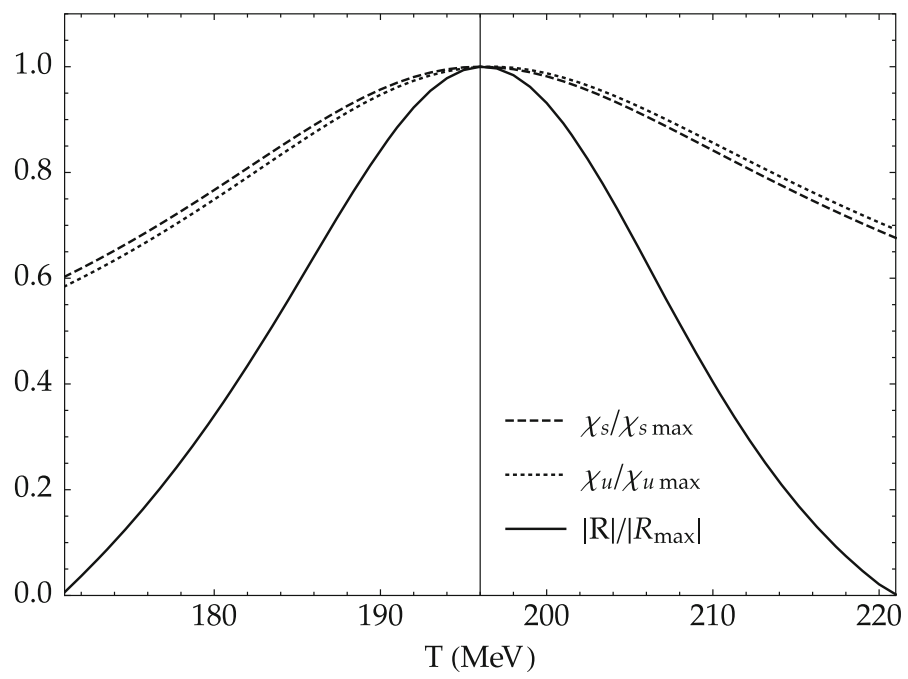

Fig. 11 The ratio $\chi_{s} / \chi_{\text {smax }}$ (dashed line), $\chi_{u} / \chi_{\text {umax }}$ (dotted line) and $|R| /|R|_{\max }$ (continuous line) at $\mu=0 \mathrm{MeV}$

In Fig. 11, the ratios $\chi_{s} / \chi_{\operatorname{smax}}$ (dashed line), $\chi_{u} / \chi_{\text {umax }}$ (dotted line) and $|R| /|R|_{\max }$ (continuous line) are depicted to visualize that the maximum in $|R|$ corresponds to the peak of chiral susceptibilities.

Figure 12 shows the transition temperature by the evaluation of $R$ : the continuous line is again obtained by the maximum of $|R|$ and the dashed ones are the spinodal curves. The black circle is at $\mu^{\star} \sim 335 \mathrm{MeV}$ and $T^{\star} \sim 35 \mathrm{MeV}$. The green band is the region of negative $R$.

\subsection{Thermal geometric definition of the phase transitions in NJL model: summary}

It is useful to conclude this section by summarizing the geometrical definition of the phase transitions:

- a second-order phase transition occurs for two flavors in the chiral limit $(m=0)$ at low chemical potential. This transition is characterized by a divergent scalar curvature;

- for chiral masses, there is a crossover, both for two and three flavors, at low chemical potential and large $T$. The transition temperature is defined as the maximum of $|R|$ in the negative- $R$ region and it is in agreement with the chiral susceptibility analysis $\chi$ [38] (Eqs. (48), (84) and (85) in Appendix);

- there exists a first-order phase transition at low temperature and large $\mu$, both with two and three flavors and both in the chiral limit or with chiral masses. This transition is related with a discontinuity in $R$.

Finally, a comment on the sign of the scalar curvature is in order.

The scalar curvature brings information on the statistical natural of the particles and on the dynamical interactions. The region with $R<0$ in Figs. 6, 8, 10 and 12 indicates that the balance between NJL attractive interactions and statistical effects is dominated by the former. On the other hand, Fermi statistics is always part of the dynamics and, at large $\mu$, the statistical effects turn out to be more and more relevant, suggesting $R>0$ as in a Fermi gas in thermodynamic geometry. 


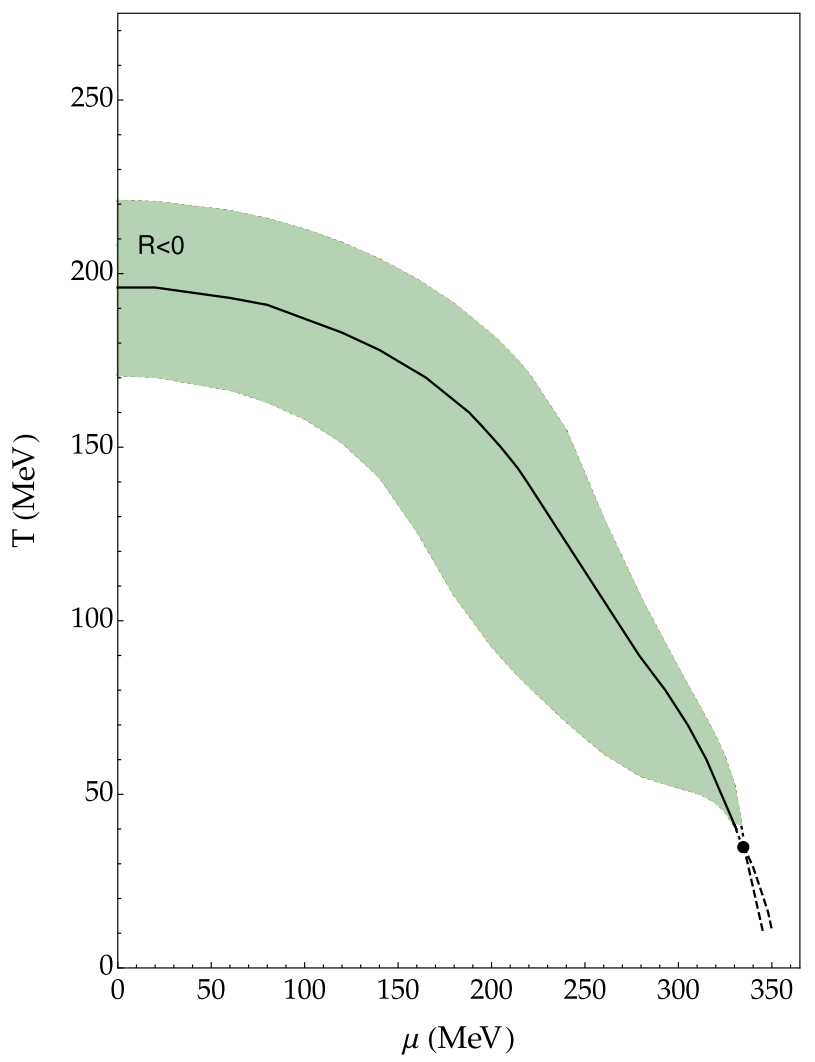

Fig. 12 The transition temperature by the $R$ conditions: continuous line is obtained by the local maximum of $|R|$, the dashed ones indicate the spinodal lines. The circle is at $\mu^{\star} \sim 335 \mathrm{MeV}$ and $T^{\star} \sim 35 \mathrm{MeV}$. The green band is the region of $R<0$

\section{NJL model and QCD crossover}

In Ref. [14], using the criterium $R=0$, the QCD deconfinement temperature has been predicted and turns out to be in agreement, within 10\%, with lattice simulations. In Sect. 4, the NJL crossover has been identified by a local maximum of $|R| \neq 0$. On the other hand, NJL model misses color confinement and, therefore, there is no a priori reason to apply the same geometric criterium.

In the thermodynamic geometry description of QCD deconfinement transition, at low baryon density [14], the criterium $R=0$ indicates the transition from a mostly fermionic system (as the quark-gluon plasma) to an essentially bosonic one (as the hadron resonance gas) and, as shown in Fig. 13, it exactly corresponds to the maximum of chiral susceptibility. On the other hand, in NJL model, where confinement is missing, the maximum of chiral susceptibility is obtained for a non-zero scalar curvature. This different behavior translates, in the thermodynamic geometrical description, the interplay between confinement and chiral symmetry breaking in QCD [39-42]. 


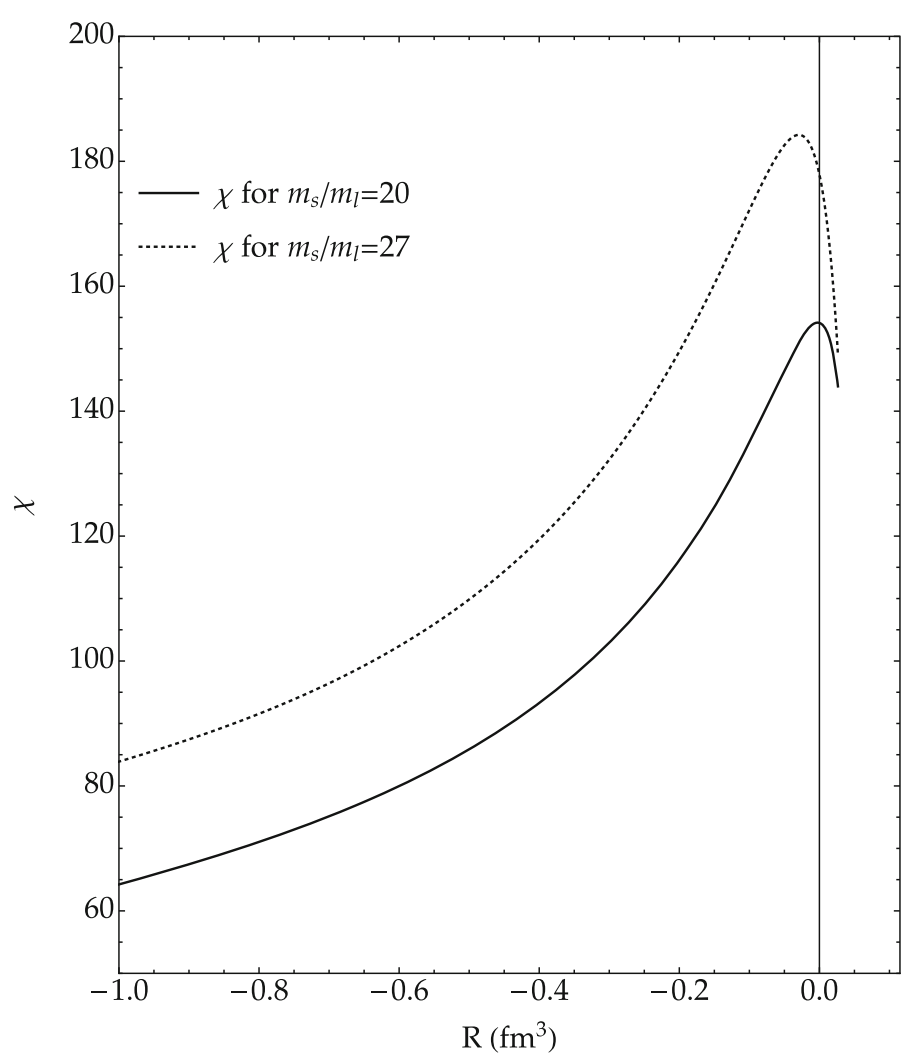

Fig. 13 The chiral susceptibility $\chi$ in QCD at $\mu=0 \mathrm{MeV}$ and as a function of the scalar curvature $R$ for physical value of the strange quark mass, $m_{s}$, and $m_{s} / m_{\ell}=20$ (dotted line) or $m_{s} / m_{\ell}=27$ (continuous line)

\section{Comments and conclusions}

For the first time thermodynamic geometry has been applied to a (although not renormalizable) field theory, with ab initio calculations. Indeed, in the previous analyses [14] for the prediction of the deconfinement temperature, Lattice data for the QCD pressure and the Hadron resonance gas models have been used.

The phase diagram in NJL model has been evaluated on the basis of the thermodynamic metric and of the corresponding scalar curvature, $R$, which contains not only the second derivatives but also higher order and mixed derivatives (up to third order). The "sensitivity" to the phase transition, naturally contained in the cumulants, does not automatically imply that the quantitative results are reliable: only after carrying out our specific calculations one can state if the method of thermodynamic geometry could be a useful tool for future analyses in field theory at finite temperature and density.

Our results show that thermodynamic geometry reliably describes the phase diagram of NJL model, both in the chiral limit and for finite mass, and indicates a geometrical interplay between chiral symmetry restoration/breaking and deconfinement/confinement regimes, as discussed in Sect. 5. 
Moreover, in a very recent paper [43], the chiral phase transition temperature $T_{c}^{0}$, corresponding to a "true" chiral transition in the limit $m_{s} / m_{l} \gg 1$, turns out to be about $25 \mathrm{MeV}$ less than the pseudo-critical temperature.

Figure 13 suggests that a small variation from $m_{s} / m_{l}=20$ to $m_{s} / m_{l}=27$ changes the maximum of chiral susceptibility from $R=0$ to a finite value of $|R|$, as in NJL model. It could be possible that considering the effective chiral limit, i.e., $m_{s} / m_{l} \gg 1$ one recovers by thermodynamic geometry a "true" chiral phase transition at lower temperature, with typical scaling laws. The role of color confinement in QCD in terms of thermodynamic geometry will be discussed in different models in a forthcoming paper.

\section{A NJL model with two quarks}

To evaluate the scalar curvature $R$, one needs the derivatives of the potential $\phi$, up to third order, which can be written in terms of the dynamical generated mass $M$. Therefore, the solution of the GAP equation uniquely determines all those functions. Indeed, after a straightforward calculation, one gets (a comma indicates partial derivative)

$$
\begin{gathered}
M_{, \beta}=\frac{b_{1} M}{1-f_{1}-f_{2} M^{2}}, \\
M_{, \gamma}=\frac{g_{1} M}{1-f_{1}-f_{2} M^{2}}, \\
M_{, \beta \beta}=d\left[b_{3} M+\left(b_{2}+f_{1, \beta}\right) M_{, \beta}+\left(b_{4}+2 T f_{2}\right) M^{2} M_{, \beta}+f_{3} M M_{, \beta}^{2}\right], \\
M_{, \gamma \gamma}=d\left[g_{3} M+\left(g_{2}+f_{1, \gamma}\right) M_{, \gamma}+g_{4} M^{2} M_{, \gamma}++f_{3} M M_{, \gamma}^{2}\right], \\
M_{, \beta \gamma}=d\left[g_{5} M+b_{2} M_{, \gamma}+f_{1, \gamma} M_{, \beta}+g_{4} M^{2} M_{, \beta}+f_{2} T M^{2} M_{, \gamma}+f_{3} M M_{, \beta} M_{, \gamma}\right]
\end{gathered}
$$

with

$$
\begin{gathered}
d=\left(1-f_{1}-f_{2} M^{2}\right)^{-1}, \\
f_{1}=\kappa_{M} \int_{0}^{\Lambda} \mathrm{d} p \frac{p^{4} \Psi}{E^{3}}, \\
f_{2}=\kappa_{M} \int_{0}^{\Lambda} \mathrm{d} p p^{2} \frac{n_{-}\left(1-n_{-}\right)+n_{+}\left(1-n_{+}\right)}{T E^{2}}, \\
f_{3}=\kappa_{M} \int_{0}^{\Lambda} \mathrm{d} p p^{4} \frac{n_{-}\left(1-n_{-}\right)+n_{+}\left(1-n_{+}\right)}{T E^{4}}, \\
b_{1}=\kappa_{M} \int_{0}^{\Lambda} \mathrm{d} p p^{2}\left[n_{-}\left(1-n_{-}\right)+n_{+}\left(1-n_{+}\right)\right], \\
b_{3}=\kappa_{M} \int_{0}^{\Lambda} \mathrm{d} p p^{2}\left[n_{-, \beta}\left(1-2 n_{-}\right)+n_{+, \beta}\left(1-2 n_{+}\right)\right], \\
b_{4}=\kappa_{M} \int_{0}^{\Lambda} \mathrm{d} p p^{2} \frac{n_{-, \beta}\left(1-2 n_{-}\right)+n_{+, \beta}\left(1-2 n_{+}\right)}{T E^{2}},
\end{gathered}
$$




$$
\begin{gathered}
g_{1}=\kappa_{M} \int_{0}^{\Lambda} \mathrm{d} p p^{2} \frac{n_{-}\left(1-n_{-}\right)-n_{+}\left(1-n_{+}\right)}{E}, \\
g_{2}=\kappa_{M} \int_{0}^{\Lambda} \mathrm{d} p p^{4} \frac{\Psi_{\gamma}}{E^{3}} \\
g_{3}=\kappa_{M} \int_{0}^{\Lambda} \mathrm{d} p p^{2} \frac{n_{-, \gamma}\left(1-2 n_{-}\right)-n_{+, \gamma}\left(1-2 n_{+}\right)}{E}, \\
g_{4}=\kappa_{M} \int_{0}^{\Lambda} \mathrm{d} p p^{2} \frac{n_{-, \gamma}\left(1-2 n_{-}\right)+n_{+, \gamma}\left(1-2 n_{+}\right)}{T E^{2}}, \\
g_{5}=\kappa_{M} \int_{0}^{\Lambda} \mathrm{d} p p^{2}\left[n_{-, \gamma}\left(1-2 n_{-}\right)+n_{+, \gamma}\left(1-2 n_{+}\right)\right], \\
\kappa_{M}=2 G \frac{N_{\mathrm{c}} N_{\mathrm{f}}}{\pi^{2}}
\end{gathered}
$$

and $n \pm$ in Eq. (16).

By deriving Eqs. (24) and (11) and defining

$$
\kappa_{\Omega}=\frac{\kappa_{M}}{2 G},
$$

one gets

$$
\begin{gathered}
\phi_{, \beta}=\kappa_{\Omega} \int_{0}^{\Lambda} \mathrm{d} p p^{2} E \Psi-\frac{(M-m)^{2}}{4 G}, \\
\phi_{, \gamma}=\kappa_{\Omega} \int_{0}^{\Lambda} \mathrm{d} p p^{2}\left(n_{+}-n_{-}\right) .
\end{gathered}
$$

The calculation of second- and third-order derivatives is straightforward.

Finally, the two-flavor chiral susceptibility, $\chi$, is defined as [38]

$$
\chi^{2 f}=\frac{\partial M}{\partial m}=\frac{1}{1-f_{1}-f_{2} M^{2}}=\frac{M_{, \beta}}{b_{1} M}=\frac{M_{, \gamma}}{g_{1} M} .
$$

\section{B Three flavors}

In three-flavor systems, the derivatives of the dynamically generated mass $M_{u}=M_{d}$ and $M_{s}$ are

$$
\begin{aligned}
& \left\{\begin{array}{l}
M_{u, \beta}\left(\delta-b_{u} M_{u} \epsilon\right)=a_{u} \epsilon-M_{s, \beta} \zeta \\
M_{s, \beta}=\frac{\left(a_{s} \theta-a_{u} \lambda\right)\left(\delta-b_{u} M_{u} \epsilon\right)-a_{u} \epsilon b_{u} \lambda M_{u}}{\left(\eta-b_{s} M_{s} \theta\right)\left(\delta-b_{u} M_{u} \epsilon\right)-b_{u} \lambda M_{u} \zeta}
\end{array}\right. \\
& \left\{\begin{array}{l}
M_{u, \gamma}\left(\delta-b_{u} M_{u} \epsilon\right)=c_{u} \epsilon-M_{s, \gamma} \zeta \\
M_{s, \gamma}=\frac{\left(c_{s} \theta-c_{u} \lambda\right)\left(\delta-b_{u} M_{u} \epsilon\right)-c_{u} \epsilon b_{u} \lambda M_{u}}{\left(\eta-b_{s} M_{s} \theta\right)\left(\delta-b_{u} M_{u} \epsilon\right)-b_{u} \lambda M_{u} \zeta}
\end{array}\right.
\end{aligned}
$$




$$
\begin{aligned}
& \left\{\begin{array}{l}
M_{u, \beta \beta}\left(\delta-b_{u} M_{u} \epsilon\right)=d_{u} \epsilon+A_{u, \beta} \epsilon, \beta-\left(M_{u, \beta} \delta_{, \beta}+M_{s, \beta} \zeta_{, \beta}+\epsilon D_{u, \beta} M_{u} M_{u, \beta}\right)-M_{s, \beta \beta} \zeta \\
M_{s, \beta \beta}=\frac{\left(d_{s} \theta-d_{u} \lambda+A_{s, \beta} \theta, \beta-A_{u, \beta} \lambda, \beta+\lambda D_{u, \beta} M_{u} M_{u, \beta}-\theta D_{s, \beta} M_{s} M_{s, \beta}\right)\left(\delta-b_{u} M_{u} \epsilon\right)}{\left(\eta-b_{s} M_{s} \theta\right)\left(\delta-b_{u} M_{u} \epsilon\right)-\zeta \lambda b_{u} M_{u}} \\
-\lambda b_{u} M_{u} \frac{\left[d_{u} \epsilon+A_{u, \beta} \epsilon, \beta-\left(M_{u, \beta} \delta_{, \beta}+M_{s, \beta} \zeta_{, \beta}+\epsilon D_{u, \beta} M_{u} M_{u, \beta}\right)\right]}{\left(\eta-b_{s} M_{s} \theta\right)\left(\delta-b_{u} M_{u} \epsilon\right)-\zeta \lambda b_{u} M_{u}}
\end{array},\right. \\
& \left\{\begin{array}{c}
\begin{array}{c}
M_{u, \gamma \gamma}\left(\delta-b_{u} M_{u} \epsilon\right)=e_{u} \epsilon+A_{u, \gamma} \epsilon_{, \gamma}-\left(M_{u, \gamma} \delta_{, \gamma}+M_{s, \gamma} \zeta_{, \gamma}+\epsilon D_{u, \gamma} M_{u} M_{u, \gamma}\right)-M_{s, \gamma \gamma} \zeta \\
M_{s, \gamma \gamma}=\frac{\left(e_{s} \theta-e_{u} \lambda+A_{s, \gamma} \theta_{, \gamma}-A_{u, \gamma} \lambda_{, \gamma}+\lambda D_{u, \gamma} M_{u} M_{u, \gamma}-\theta D_{s, \gamma} M_{s} M_{s, \gamma}\right)\left(\delta-b_{u} M_{u} \epsilon\right)}{\left(\eta-b_{s} M_{s} \theta\right)\left(\delta-b_{u} M_{u} \epsilon\right)-\zeta \lambda b_{u} M_{u}} \\
-\lambda b_{u} M_{u} \frac{\left[e_{u} \epsilon+A_{u, \gamma} \epsilon_{, \gamma}-\left(M_{u, \gamma} \delta_{, \gamma}+M_{s, \gamma} \zeta_{, \gamma}+\epsilon D_{u, \gamma} M_{u} M_{u, \gamma}\right)\right]}{\left(\eta-b_{s} M_{s} \theta\right)\left(\delta-b_{u} M_{u} \epsilon\right)-\zeta \lambda b_{u} M_{u}}
\end{array}
\end{array}\right.
\end{aligned}
$$

and

$$
\left\{\begin{array}{c}
M_{u, \beta \gamma}\left(\delta-b_{u} M_{u} \epsilon\right)=f_{u} \epsilon+A_{u, \beta} \epsilon_{, \gamma}-\left(M_{u, \beta} \delta_{, \gamma}+M_{s, \beta} \zeta_{, \gamma}+\epsilon D_{u, \beta} M_{u} M_{u, \gamma}\right)-M_{s, \beta \gamma} \zeta \\
M_{s, \beta \gamma}=\frac{\left(f_{s} \theta-f_{u} \lambda+A_{s, \beta} \theta_{, \gamma}-A_{u, \beta} \lambda, \gamma+\lambda D_{u, \beta} M_{u} M_{u, \gamma}-\theta D_{s, \beta} M_{s} M_{s, \gamma}\right)\left(\delta-b_{u} M_{u} \epsilon\right)}{\left(\eta-b_{s} M_{s} \theta\right)\left(\delta-b_{u} M_{u} \epsilon\right)-\zeta \lambda b_{u} M_{u}} \\
-\lambda b_{u} M_{u} \frac{\left[f_{u} \epsilon+A_{u, \beta} \epsilon_{, \gamma}-\left(M_{u, \beta} \delta_{, \gamma}+M_{s, \beta} \zeta_{, \gamma}+\epsilon D_{u, \beta} M_{u} M_{u, \gamma}\right)\right]}{\left(\eta-b_{s} M_{s} \theta\right)\left(\delta-b_{u} M_{u} \epsilon\right)-\zeta \lambda b_{u} M_{u}}
\end{array}\right.
$$

where

$$
\begin{aligned}
& a_{f}=\frac{N_{\mathrm{c}}}{\pi^{2}} \int_{0}^{\Lambda} \mathrm{d} p p^{2}\left[n_{-f}\left(1-n_{-f}\right)+n_{+f}\left(1-n_{+f}\right)\right] \text {, } \\
& b_{f}=\frac{N_{\mathrm{c}}}{\pi^{2}} \int_{0}^{\Lambda} \mathrm{d} p p^{2} \frac{n_{-f}\left(1-n_{-f}\right)+n_{+f}\left(1-n_{+f}\right)}{T E_{f}^{2}} \\
& c_{f}=\frac{N_{\mathrm{c}}}{\pi^{2}} \int_{0}^{\Lambda} \mathrm{d} p p^{2} \frac{n_{-f}\left(1-n_{-f}\right)-n_{+f}\left(1-n_{+f}\right)}{E_{f}}, \\
& d_{f}=\frac{N_{\mathrm{c}}}{\pi^{2}} \int_{0}^{\Lambda} \mathrm{d} p p^{2}\left\{\left(\frac{2 M M_{, \beta}}{E^{2}}+p^{2} \frac{M_{, \beta}^{2}}{T E^{4}}\right)\right. \\
& \times\left[n_{-f}\left(1-n_{-f}\right)+n_{+f}\left(1-n_{+f}\right)\right] \\
& \left.+\left(1+\frac{M M_{, \beta}}{T E^{2}}\right)\left[\left(1-2 n_{-f}\right) n_{-f, \beta}+\left(1-2 n_{+f}\right) n_{+f, \beta}\right]\right\} \text {, } \\
& e_{f}=\frac{N_{\mathrm{c}}}{\pi^{2}} \int_{0}^{\Lambda} \mathrm{d} p p^{2}\left\{p^{2} \frac{M_{, \gamma}^{2}}{T E^{4}}\left[n_{-f}\left(1-n_{-f}\right)+n_{+f}\left(1-n_{+f}\right)\right]\right. \\
& +\frac{M M_{, \gamma}}{T E^{2}}\left[\left(1-2 n_{-f}\right) n_{-f, \gamma}+\left(1-2 n_{+f}\right) n_{+f, \gamma}\right] \\
& \left.+\frac{\left(1-2 n_{-f}\right) n_{-f, \gamma}-\left(1-2 n_{+f}\right) n_{+f, \gamma}}{E}\right\} \text {, } \\
& f_{f}=\frac{N_{\mathrm{c}}}{\pi^{2}} \int_{0}^{\Lambda} \mathrm{d} p p^{2}\left\{\left(\frac{M M_{, \gamma}}{E^{2}}+p^{2} \frac{M_{, \beta} M_{, \gamma}}{T E^{4}}\right)\right.
\end{aligned}
$$




$$
\begin{aligned}
& \times\left[n_{-f}\left(1-n_{-f}\right)+n_{+f}\left(1-n_{+f}\right)\right] \\
& \left.+\left(1+\frac{M M_{, \beta}}{T E^{2}}\right)\left[\left(1-2 n_{-f}\right) n_{-f, \gamma}+\left(1-2 n_{+f}\right) n_{+f, \gamma}\right]\right\}, \\
& A_{f, \beta}=a_{f}+b_{f} M_{f} M_{f, \beta}, \\
& A_{f, \gamma}=c_{f}+b_{f} M_{f} M_{f, \gamma}, \\
& C_{f, \beta \beta}=d_{f}+b_{f} M_{f} M_{f, \beta \beta}, \\
& C_{f, \gamma \gamma}=e_{f}+b_{f} M_{f} M_{f, \gamma \gamma} \text {, } \\
& C_{f, \beta \gamma}=f_{f}+b_{f} M_{f} M_{f, \beta \gamma}, \\
& B\left(M_{u}, M_{s}\right)=4 G-2 \frac{K^{2}}{G} u^{2}-2 K s, \\
& \delta\left(M_{u}, M_{s}\right)=\left(1-F_{1 u} B\right), \\
& \zeta\left(M_{u}, M_{s}\right)=2 K u, \\
& \epsilon\left(M_{u}, M_{s}\right)=B M_{u}, \\
& \eta\left(M_{u}, M_{s}\right)=\left(1-4 G F_{1 s}\right)\left(1-F_{1 u} B\right)-8 K^{2} u^{2} F_{1 u}, \\
& \theta\left(M_{u}, M_{s}\right)=4 G\left(1-F_{1 u} B\right) M_{s}, \\
& \lambda\left(M_{u}, M_{s}\right)=4 K u M_{u}, \\
& F_{1 f}=\frac{N_{\mathrm{c}}}{\pi^{2}} \int_{0}^{\Lambda} \mathrm{d} p p^{4} \frac{\Psi_{f}}{E_{f}^{3}}, \\
& n_{f \pm}=\frac{1}{1+\exp \left\{\frac{\sqrt{p^{2}+M_{f}^{2}} \pm \mu_{f}}{T}\right\}}
\end{aligned}
$$

and $u \equiv\langle\bar{u} u\rangle, s \equiv\langle\bar{s} s\rangle$.

About the thermodynamic potential $\phi=-\Omega \beta$, one has

$$
\begin{aligned}
\phi, \beta= & \sum_{f=u, d, s} \frac{N_{\mathrm{c}}}{\pi^{2}} \int_{0}^{\Lambda} \mathrm{d} p p^{2} E_{f} \Psi_{f}+2 G s^{2} \\
& +u\left(M_{u}-m_{u}\right)+s\left(M_{s}-m_{s}\right) \\
= & \sum_{f=u, d, s} \frac{N_{\mathrm{c}}}{\pi^{2}} \int_{0}^{\Lambda} \mathrm{d} p p^{2} E_{f} \Psi_{f}+K u^{2} s \\
& +u\left(M_{u}-m_{u}\right)+\frac{s\left(M_{s}-m_{s}\right)}{2}, \\
\phi_{, \gamma}= & \sum_{f=u, d, s} \frac{N_{\mathrm{c}}}{\pi^{2}} \int_{0}^{\Lambda} \mathrm{d} p p^{2}\left(n_{+f}-n_{-f}\right) \\
\phi_{, \beta \beta}= & -\sum_{f=u, d, s} \frac{N_{\mathrm{c}}}{\pi^{2}} \int_{0}^{\Lambda} \mathrm{d} p p^{2} E_{f}\left(n_{+f, \beta}+n_{-f, \beta}\right) \\
\phi_{, \beta \gamma}= & \sum_{f=u, d, s} \frac{N_{\mathrm{c}}}{\pi^{2}} \int_{0}^{\Lambda} \mathrm{d} p p^{2}\left(n_{+f, \beta}-n_{-f, \beta}\right) \\
\phi_{, \gamma \gamma}= & \sum_{f=u, d, s} \frac{N_{\mathrm{c}}}{\pi^{2}} \int_{0}^{\Lambda} \mathrm{d} p p^{2}\left(n_{+f, \gamma}-n_{-f, \gamma}\right)
\end{aligned}
$$




$$
\begin{aligned}
\phi_{, \beta \beta \gamma}= & \sum_{f=u, d, s} \frac{N_{\mathrm{c}}}{\pi^{2}} \int_{0}^{\Lambda} \mathrm{d} p p^{2}\left(n_{+f, \beta \beta}-n_{-f, \beta \beta}\right) \\
\phi_{, \beta \gamma \gamma}= & \sum_{f=u, d, s} \frac{N_{\mathrm{c}}}{\pi^{2}} \int_{0}^{\Lambda} \mathrm{d} p p^{2}\left(n_{+f, \beta \gamma}-n_{-f, \beta \gamma}\right) \\
\phi_{, \gamma \gamma \gamma}= & \sum_{f=u, d, s} \frac{N_{\mathrm{c}}}{\pi^{2}} \int_{0}^{\Lambda} \mathrm{d} p p^{2}\left(n_{+f, \gamma \gamma}-n_{-f, \gamma \gamma}\right) \\
\phi_{, \beta \beta \beta}= & -\sum_{f=u, d, s} \frac{N_{\mathrm{c}}}{\pi^{2}} \int_{0}^{\Lambda} \mathrm{d} p p^{2} E_{f}\left(n_{+f, \beta \beta}+n_{-f, \beta \beta}\right) \\
& +\sum_{f=u, d, s}\left(a_{f}+b_{f} M_{f} M_{f, \beta}\right) M_{f} M_{f, \beta} .
\end{aligned}
$$

Finally, by defining

$$
H_{f}=F_{1 f}+b_{f} M_{f}^{2}
$$

the chiral susceptibilities are

$$
\chi_{u}=\chi_{d}=\frac{\partial M_{u}}{\partial m_{u}}=\frac{1-4 G H_{s}}{1-4 G\left(H_{u}+H_{s}\right)+4 H_{u} H_{s}\left(4 G^{2}-2 K G s-K^{2} u^{2}\right)+2 K s H_{u}}
$$

and

$$
\chi_{s}=\frac{\partial M_{s}}{\partial m_{s}}==\frac{1-(4 G-2 K s) H_{u}}{1-4 G\left(H_{u}+H_{s}\right)+4 H_{u} H_{s}\left(4 G^{2}-2 K G s-2 K^{2} u^{2}\right)+2 K s H_{u}} .
$$

\section{References}

1. N. Ay, J. Jost, H. Van Le, L. Schwachhofer, Information Geometry, A Series of Modern Surveys in Mathematics, vol. 64 (Springer, Berlin, 2017)

2. S. Amari, Information Geometry and Its Applications, Applied Mathematical Sciences, vol. 194 (Springer, Berlin, 2016)

3. M. Suzuki, Information geometry and statistical manifold. arXiv:1410.3369

4. R.C. Rao, Information and accuracy attainable in the estimation of statistical parameters. Bull. Calcutta Math. Soc. 37, 81-91 (1945)

5. F. Weinhold, Metric geometry of equilibrium thermodynamics. J. Chem. Phys. 63, 2479, 2484, 2488, 2496 (1975)

6. George Ruppeiner, Thermodynamics: a Riemannian geometric model. Phys. Rev. A 20, 1608-1613 (1979)

7. George Ruppeiner, Riemannian geometry in thermodynamic fluctuation theory. Rev. Mod. Phys. 67, 605-659 (1995). [Erratum: Rev. Mod. Phys. 68, 313 (1996)]

8. George Ruppeiner, Anurag Sahay, Tapobrata Sarkar, Gautam Sengupta, Thermodynamic geometry, phase transitions, and the Widom line. Phys. Rev. E 86, 052103 (2012)

9. Helge-Otmar May, Peter Mausbach, Riemannian geometry study of vapor-liquid phase equilibria and supercritical behavior of the Lennard-Jones fluid. Phys. Rev. E 85, 031201 (2012)

10. Helge-Otmar May, Peter Mausbach, George Ruppeiner, Thermodynamic curvature for attractive and repulsive intermolecular forces. Phys. Rev. E 88, 032123 (2013)

11. Anshuman Dey, Pratim Roy, Tapobrata Sarkar, Information geometry, phase transitions, and the Widom line: magnetic and liquid systems. Phys. A 392, 6341-6352 (2013)

12. Pankaj Chaturvedi, Anirban Das, Gautam Sengupta, Thermodynamic geometry and phase transitions of dyonic charged AdS black holes. Eur. Phys. J. C 77(2), 110 (2017) 
13. Anurag Sahay, Rishabh Jha, Geometry of criticality, supercriticality and Hawking-Page transitions in Gauss-Bonnet-AdS black holes. Phys. Rev. D 96(12), 126017 (2017)

14. P. Castorina, M. Imbrosciano, D. Lanteri, Thermodynamic geometry of strongly interacting matter. Phys. Rev. D 98(9), 096006 (2018)

15. Paolo Castorina, Mauro Imbrosciano, Daniele Lanteri, Thermodynamic geometry and deconfinement temperature. Eur. Phys. J. Plus 134(4), 164 (2019)

16. George Ruppeiner, Riemannian geometric approach to critical points: general theory. Phys. Rev. E 57, 5135-5145 (1998)

17. B. Widom, The critical point and scaling theory. Physica 73(1), 107-118 (1974)

18. George Ruppeiner, Thermodynamic curvature from the critical point to the triple point. Phys. Rev. E 86, 021130 (2012)

19. H. Janyszek, R. Mrugala, Riemannian geometry and the thermodynamics of model magnetic systems. Phys. Rev. A 39, 6515-6523 (1989)

20. H. Janyszek, R. Mrugala, Riemannian and finslerian geometry and fluctuations of thermodynamic systems. Advances in Thermodynamics, vol. 3. Nonequilibrium Theory and Extremum Principles, pp. 159-174 (1990)

21. G. Ruppeiner, Thermodynamic curvature measures interactions. Am. J. Phys. 78, 1170 (2010)

22. Michele Floris, Hadron yields and the phase diagram of strongly interacting matter. Nucl. Phys. A 931, 103-112 (2014)

23. S. Das, Identified particle production and freeze-out properties in heavy-ion collisions at RHIC Beam Energy Scan program (2014) [EPJ Web Conf. 90, 08007 (2015)]

24. L. Adamczyk et al., Bulk properties of the medium produced in relativistic heavy-ion collisions from the beam energy scan program. Phys. Rev. C 96(4), 044904 (2017)

25. Patrick Steinbrecher, The QCD crossover at zero and non-zero baryon densities from Lattice QCD. Nucl. Phys. A 982, 847-850 (2019)

26. A. Bazavov et al., The QCD equation of state to $\mathcal{O}\left(\mu_{B}^{6}\right)$ from lattice QCD. Phys. Rev. D 95(5), 054504 (2017)

27. H. Janyszek, Riemannian geometry and stability of thermodynamical equilibrium systems. J. Phys. A Math. Gen. 23(4), 477-490 (1990)

28. M.R. Ubriaco, The role of curvature in quantum statistical mechanics. J. Phys. Conf. Ser. 766, 012007 (2016)

29. R. Ruppeiner, N. Dyjack, A. McAloon, J. Stoops, Solid-like features in dense vapors near the fluid critical point. J. Chem. Phys. 146, 224501 (2017)

30. Behrouz Mirza, Hosein Mohammadzadeh, Ruppeiner geometry of anyon gas. Phys. Rev. E 78, 021127 (2008)

31. M.R. Ubriaco, Stability and anyonic behavior of systems with m-statistics. Phys. A Stat. Mech. Appl. 392(20), 4868-4873 (2013)

32. Anurag Sahay, Tapobrata Sarkar, Gautam Sengupta, On the thermodynamic geometry and critical phenomena of AdS black holes. JHEP 07, 082 (2010)

33. G. Ruppeiner, P. Mausbach, H.-0. May, Thermodynamic r-diagrams reveal solid-like fluid states. Phys. Lett. A 379(7), 646-649 (2015)

34. P. Zhuang, J. Hufner, S.P. Klevansky, Thermodynamics of a quark-meson plasma in the Nambu-JonaLasinio model. Nucl. Phys. A 576, 525-552 (1994)

35. T.M. Schwarz, S.P. Klevansky, G. Papp, The Phase diagram and bulk thermodynamical quantities in the NJL model at finite temperature and density. Phys. Rev. C 60, 055205 (1999)

36. Michael Buballa, NJL model analysis of quark matter at large density. Phys. Rep. 407, 205-376 (2005)

37. A. Barducci, R. Casalbuoni, G. Pettini, L. Ravagli, A NJL-based study of the QCD critical line. Phys. Rev. D 72, 056002 (2005)

38. Yue Zhao, Lei Chang, Wei Yuan, Yu-xin Liu, Chiral susceptibility and chiral phase transition in NambuJona-Lasinio model. Eur. Phys. J. C 56, 483-492 (2008)

39. Aharon Casher, Chiral symmetry breaking in quark confining theories. Phys. Lett. 83B, 395-398 (1979)

40. Tom Banks, A. Casher, Chiral symmetry breaking in confining theories. Nucl. Phys. B 169, 103-125 (1980)

41. P. Cea, P. Castorina, Quark confinement and chiral symmetry breaking. Nuovo Cim. A 81, 567 (1984)

42. S. Digal, E. Laermann, H. Satz, Deconfinement through chiral symmetry restoration in two flavor QCD. Eur. Phys. J. C 18, 583-586 (2001)

43. H.T. Ding, P. Hegde, F. Karsch, A. Lahiri, S.T. Li, S. Mukherjee, P. Petreczky, Chiral phase transition of (2+1)-flavor QCD. Nucl. Phys. A 982, 211-214 (2019) 